\title{
On one-dimensional diffusion processes living in a bounded space interval
}

\author{
by Anna Milian (Kraków)
}

\begin{abstract}
We prove that under some assumptions a one-dimensional Itô equation has a strong solution concentrated on a finite spatial interval, and the pathwise uniqueness holds.
\end{abstract}

Introduction. In the present paper we will consider a diffusion satisfying the stochastic integral Itô equation

$$
X(t)=X(0)+\int_{0}^{t} a(s, X(s)) d s+\int_{0}^{t} b(s, X(s)) d W(s)
$$

where $W(t)$ is a given one-dimensional Wiener process on a probability space $(\Omega, \mathcal{F}, P)$.

It is known ([1], p. 372) that if $b\left(t, r_{i}\right)=0 \leq(-1)^{i} a\left(t, r_{i}\right), i=0,1, t \geq 0$, and if $a$ and $b$ are sufficiently regular, then (1) has a unique solution $X(t)$ concentrated on the interval $\left[r_{0}, r_{1}\right]$.

In this paper we consider strong solutions of (1) ([3], p. 149). An example of a stochastic integral equation which has a solution but has no strong solution is due to H. Tanaka ([3], p. 152). We will give some sufficient conditions in order that (1) has a unique (in the sense of pathwise uniqueness) strong solution $X(t)$, satisfying $X(t) \in(\alpha(t), \beta(t))$ for $t \geq 0$, where $\alpha$ and $\beta$ are given sufficiently regular real-valued functions defined for $t \geq 0$.

Existence and pathwise uniqueness of the strong solution of equation (1) on a finite spatial interval. First we give some sufficient conditions in order that a strong solution $X(t)$ of the stochastic equation

1991 Mathematics Subject Classification: 60H20.

Key words and phrases: one-dimensional Itô equation, bounded strong solutions, timedependent boundaries. 
(1) exists and satisfies the additional condition

$$
|X(t)|<1 \quad \text { for } t \geq 0 .
$$

We will need the following theorem ([1], Theorem 3.11, p. 300 in the case $d=1)$ :

Theorem 1. Let $a:[0, \infty) \times \mathbb{R} \rightarrow \mathbb{R}$ and $b:[0, \infty) \times \mathbb{R} \rightarrow \mathbb{R}$ be locally bounded and Borel measurable. Suppose that for each $T>0$ and $N \geq 1$ there exist constants $K_{T}$ and $K_{T, N}$ such that

1) $|b(t, x)|^{2} \leq K_{T}\left(1+x^{2}\right), \quad x a(t, x) \leq K_{T}\left(1+x^{2}\right)$,

$$
0 \leq t \leq T, x \in \mathbb{R}
$$

2) $|b(t, x)-b(t, y)| \vee|a(t, x)-a(t, y)| \leq K_{T, N}|x-y|$,

$$
0 \leq t \leq T,|x| \vee|y| \leq N .
$$

Given a 1-dimensional Brownian motion $W$ and an independent $\mathbb{R}$-valued random variable $\xi$ on a probability space $(\Omega, \mathcal{F}, P)$ such that $\mathbb{E}\left[|\xi|^{2}\right]<\infty$, there exists a process $X$ with $X(0)=\xi$ a.s. such that $\left(\Omega, \mathcal{F}, P,\left\{\mathcal{F}_{t}\right\}, W, X\right)$ is a solution of the stochastic integral equation (1), where $\mathcal{F}_{t}=\mathcal{F}_{t}^{W} \vee \sigma(\xi)$ $(\sigma(\xi)$ denotes the minimal $\sigma$-algebra with respect to which $\xi$ is measurable).

Let $\Phi(t, x)$ be a monotone (in $x)$ continuous function, defined for $t \in$ $[0, T], x \in(-1,1)$, for which the derivatives $\Phi_{t}(t, x), \Phi_{x}(t, x)$ and $\Phi_{x x}(t, x)$ exist and are continuous. For each $t \in[0, T]$ there exists a function $\Psi(t, x)$ inverse to $\Phi(t, x)$, i.e. $\Phi(t, \Psi(t, x))=x, \Psi(t, \Phi(t, x))=x$. If $\xi(t)$ satisfies (1) and $|\xi(t)|<1$ for $t \in[0, T]$, then applying Itô's formula ([2], Theorem 4, p. 24) we conclude that the process $X(t)=\Phi(t, \xi(t))$ satisfies the equation

$$
d X(t)=m(t, X(t)) d t+\sigma(t, X(t)) d W(t),
$$

where

$$
\begin{aligned}
m(t, x)= & \frac{\partial \Phi}{\partial t}(t, \Psi(t, x))+\frac{\partial \Phi}{\partial x}(t, \Psi(t, x)) a(t, \Psi(t, x)) \\
& +\frac{1}{2} \frac{\partial^{2} \Phi}{\partial x^{2}}(t, \Psi(t, x)) b^{2}(t, \Psi(t, x)), \\
\sigma(t, x)= & \frac{\partial \Phi}{\partial x}(t, \Psi(t, x)) b(t, \Psi(t, x)) .
\end{aligned}
$$

Let

$$
\begin{gathered}
p(x)=\int_{0}^{x} \frac{d s}{\sqrt{1+s^{2}}}, \\
\Phi(x)=p^{-1}\left(\ln \frac{1+x}{1-x}\right) .
\end{gathered}
$$


Note that $\Phi$ is an increasing one-to-one mapping from $(-1,1)$ onto $\mathbb{R}$. Define

$$
\Psi(x)=\Phi^{-1}(x)=\frac{e^{p(x)}-1}{e^{p(x)}+1} .
$$

TheOREM 2. Assume that a 1-dimensional Wiener process $W(t)$ and an independent $\mathbb{R}$-valued random variable $X_{0}$ on a probability space $(\Omega, \mathcal{F}, P)$ are given, $\left|X_{0}\right|<1$ with probability 1 . Let the coefficients $a(t, x)$ and $b(t, x)$ of (1) be defined, Borel measurable and locally bounded for $t \geq 0,|x| \leq 1$. Suppose further that

1) for each $T>0$ there exists a constant $K_{T}$ such that

$$
|a(t, x)-a(t, y)|+|b(t, x)-b(t, y)| \leq K_{T}|x-y|
$$

$t \in[0, T],|x| \leq 1,|y| \leq 1$,

2) $b(t, \mp 1)=0$ for $0 \leq t \leq T$,

3) $a(t, 1) \leq 0, a(t,-1) \geq 0$ for $0 \leq t \leq T$,

4) $\mathbb{E}\left(\Phi\left(X_{0}\right)\right)^{2}<\infty$.

Then there exists a process $X(t)$ with $X(0)=X_{0}$ a.s. such that $(\Omega, \mathcal{F}, P$, $\left.\left\{\mathcal{F}_{t}\right\}, W, X(t)\right)$ is a solution of the stochastic integral equation (1), where $\mathcal{F}_{t}=\mathcal{F}_{t}^{W} \vee \sigma\left(X_{0}\right)$, and $|X(t)|<1$ for $0 \leq t \leq T$ a.s. If $X_{1}(t)$ and $X_{2}(t)$ are two solutions of $(1)$ with $P\left(X_{i}(0)=X_{0}\right)=1$ and $\left|X_{i}(t)\right|<1$ a.s. for $i=1,2$ and for $t \in[0, T]$, then

$$
P\left\{\sup _{0 \leq t \leq T}\left|X_{1}(t)-X_{2}(t)\right|=0\right\}=1 .
$$

Proof. By 1) and 2) we have $|b(t, x)|=|b(t, x)-b(t, 1)| \leq K_{T}|x-1|$. Thus

$$
\left|\frac{b(t, x)}{x-1}\right| \leq K_{T} \quad \text { for } 0 \leq t \leq T,|x|<1
$$

Analogously

$$
\left|\frac{b(t, x)}{x+1}\right| \leq K_{T} \quad \text { for } 0 \leq t \leq T,|x|<1 .
$$

From 1) and 3) we have

$$
\begin{aligned}
\frac{a(t, x)}{x+1} & =\frac{a(t, x)-a(t,-1)}{x+1}+\frac{a(t,-1)}{x+1} \\
& \geq \frac{a(t, x)-a(t,-1)}{x+1} \geq \frac{-|a(t, x)-a(t,-1)|}{x+1} .
\end{aligned}
$$

Hence

$$
\frac{a(t, x)}{x+1} \geq-K_{T} \quad \text { for } 0 \leq t \leq T,|x|<1 .
$$


Analogously

$$
\frac{a(t, x)}{1-x} \leq \frac{a(t, x)-a(t, 1)}{1-x} \leq K_{T} \quad \text { for } 0 \leq t \leq T,|x|<1 .
$$

Consider the equation (1) with the drift coefficient $m(t, x)$ and the diffusion coefficient $\sigma(t, x)$ given by the formulas (2) and (3); $\Phi$ and $\Psi$ are given by (5) and (6). We will prove that they satisfy all assumptions of Theorem 1. By (6)

$$
\begin{aligned}
\Psi^{\prime}(x) & =\frac{2 e^{p(x)}}{\sqrt{1+x^{2}}\left(e^{p(x)}+1\right)^{2}} \\
\Psi^{\prime \prime}(x) & =\frac{2 e^{p(x)}\left[\left(1-e^{p(x)}\right) \sqrt{1+x^{2}}-x\left(e^{p(x)}+1\right)\right]}{\left(1+x^{2}\right)^{3 / 2}\left[e^{p(x)}+1\right]^{3}} .
\end{aligned}
$$

Since $\Phi \circ \Psi=$ id, we have

$$
\Phi^{\prime}(\Psi(x))=\frac{\sqrt{1+x^{2}}\left(e^{p(x)}+1\right)^{2}}{2 e^{p(x)}} .
$$

Differentiating the identity $\Phi^{\prime}(\Psi(x)) \Psi^{\prime}(x)=1$, we obtain $\Phi^{\prime \prime}(\Psi(x))=$ $-\Psi^{\prime \prime}(x)\left\{\Psi^{\prime}(x)\right\}^{-3}$. Thus

$$
\begin{aligned}
m(t, x)= & a(t, \Psi(x)) \frac{\sqrt{1+x^{2}}\left(e^{p(x)}+1\right)^{2}}{2 e^{p(x)}} \\
& -\frac{1}{2} b^{2}(t, \Psi(x))\left(\frac{b(t, \Psi(x))}{\Psi^{\prime}(x)}\right)^{2} \frac{\Psi^{\prime \prime}(x)}{\Psi^{\prime}(x)}, \\
\sigma(t, x)= & b(t, \Psi(x)) \frac{\sqrt{1+x^{2}}\left(e^{p(x)}+1\right)^{2}}{2 e^{p(x)}} .
\end{aligned}
$$

If $x \geq 0$, then $p(x) \geq 0$ and by (7) and (12) we obtain

$$
\begin{aligned}
|\sigma(t, x)| & =\left|\frac{b(t, \Psi(x))}{1-\Psi(x)}\right||1-\Psi(x)| \frac{\sqrt{1+x^{2}}\left(e^{p(x)}+1\right)^{2}}{2 e^{p(x)}} \\
& \leq K_{T} \frac{e^{p(x)}+1}{e^{p(x)}} \sqrt{1+x^{2}} \leq 2 K_{T} \sqrt{1+x^{2}} .
\end{aligned}
$$

If $x \leq 0$, then $p(x) \leq 0$ and by (8) and (12) we have

$$
\begin{aligned}
|\sigma(t, x)| & =\left|\frac{b(t, \Psi(x))}{1+\Psi(x)}\right||1+\Psi(x)| \frac{\sqrt{1+x^{2}}\left(e^{p(x)}+1\right)^{2}}{2 e^{p(x)}} \\
& \leq K_{T} \sqrt{1+x^{2}}\left(e^{p(x)}+1\right) \leq 2 K_{T} \sqrt{1+x^{2}} .
\end{aligned}
$$

Thus $\sigma(t, x)$ satisfies Condition 1$)$ of Theorem 1 . 
If $x \geq 0$, then by (10)

$$
\begin{aligned}
x a(t, \Psi(x)) \frac{\sqrt{1+x^{2}}\left(e^{p(x)}+1\right)^{2}}{2 e^{p(x)}} & =\frac{a(t, \Psi(x))}{1-\Psi(x)} x \sqrt{1+x^{2}}\left(1+e^{-p(x)}\right) \\
& \leq 2 K_{T}\left(1+x^{2}\right) .
\end{aligned}
$$

If $x \leq 0$, then by (9)

(14) $\quad x a(t, \Psi(x)) \frac{\sqrt{1+x^{2}}\left(e^{p(x)}+1\right)^{2}}{2 e^{p(x)}}=\frac{a(t, \Psi(x))}{1+\Psi(x)} x \sqrt{1+x^{2}}\left(e^{p(x)}+1\right)$

$\leq-K_{T} x \sqrt{1+x^{2}}\left(e^{p(x)}+1\right)=K_{T}(-x) \sqrt{1+x^{2}}\left(e^{p(x)}+1\right) \leq 2 K_{T}\left(1+x^{2}\right)$.

Next

$$
-\frac{1}{2} \frac{\Psi^{\prime \prime}(x)}{\Psi^{\prime}(x)}=\frac{1}{2} \frac{\Psi(x)}{\sqrt{1+x^{2}}}+\frac{x}{2\left(1+x^{2}\right)} .
$$

Since $b(t, \Psi(x)) / \Psi^{\prime}(x)=\sigma(t, x)$ satisfies Condition 1) of Theorem 1, by (13)-(15) we conclude that $m(t, x)$ satisfies Condition 1) of Theorem 1. Condition 2) of Theorem 1 also holds.

Thus, there exists a process $Y(t)$ satisfying (1) with the coefficients $m(t, x)$ and $\sigma(t, x)$ with the initial condition $Y(0)=\Phi\left(0, X_{0}\right)$. Using Itô's formula, we prove that the process $X(t)=\Psi(t, Y(t))$ satisfies the equation

$$
\begin{aligned}
d X(t) & =a_{1}(t, X(t)) d t+b_{1}(t, X(t)) d W(t), \quad \text { where } \\
a_{1}(t, x) & =\Psi^{\prime}(\Phi(x)) m(t, \Phi(x))+\frac{1}{2} \Psi^{\prime \prime}(\Phi(x)) \sigma^{2}(t, \Phi(x)), \\
b_{1}(t, x) & =\Psi^{\prime}(\Phi(x)) \sigma(t, \Phi(x)) .
\end{aligned}
$$

Applying formulas (2), (3) and the identity $\Psi \circ \Phi=\mathrm{id}$, we obtain

$$
a_{1}(t, x)=a(t, x)(\Psi \circ \Phi)^{\prime}(x)+\frac{1}{2} b^{2}(t, x)(\Psi \circ \Phi)^{\prime \prime}(x)=a(t, x) .
$$

Analogously,

$$
b_{1}(t, x)=b(t, x)(\Psi \circ \Phi)^{\prime}(x)=b(t, x) .
$$

Thus $X(t)$ is a strong solution of (1) with the initial condition $X(0)=$ $\Psi(0, Y(0))=\Psi\left(0, \Phi\left(0, X_{0}\right)\right)=X_{0}$. Moreover, $|X(t)|<1$ for $t \geq 0$ a.s. Let $X_{1}(t)$ and $X_{2}(t)$ be two solutions of (1) with $P\left(X_{i}(0)=X_{0}\right)=1$ and $\left|X_{i}(t)\right|<1$ for $t \in[0, T], i=1,2$. Extend $b$ to be zero outside $[-1,1]$ and set $a(t, x)=a(t,-1), x<-1$, and $a(t, x)=a(t, 1), x>1$. Then from Theorem 3.7 of [1], p. 297, we conclude that $P\left\{X_{1}(t)=X_{2}(t)\right.$ for $0 \leq t \leq T\}=1$, that is to say, the pathwise uniqueness holds. The proof is finished.

If the coefficients of (1) satisfy the assumptions of Theorem 2 and additionally $a(t, x)$ and $b(t, x)$ are continuous in both arguments, then ([2], Theorem 2, p. 68 and [2], p. 66) the solution of (1) is a diffusion with diffusion coefficient $b^{2}(t, x)$ and drift coefficient $a(t, x)$. 
Let $f(t, x)$ be a real function defined in $G=\{(t, x): 0 \leq t \leq T, \alpha(t) \leq$ $x \leq \beta(t)\}$, where $\alpha, \beta \in C^{1}[0, T]$. Assume that $f(t, x)$ is $C^{3}$ in some open neighbourhood of $G$ and $(\partial f / \partial x)(t, x)>0$ in $G$. Moreover, suppose $f(t, \cdot)$ is a one-to-one mapping from $(\alpha(t), \beta(t))$ onto $(-1,1)$ for $t \in[0, T]$. Let $g(t, \cdot)$ denote the inverse of $f(t, \cdot)$, i.e.,

$$
g(t, f(t, x)) \equiv x \equiv f(t, g(t, x)) \quad \text { for } t \in[0, T] .
$$

From Theorem 2 follows:

COROllary 1. Assume that a 1-dimensional Wiener process $W(t)$ and an independent $\mathbb{R}$-valued random variable $X_{0}$ on a probability space $(\Omega$, $\mathcal{F}, P)$ are given, and $X_{0} \in(\alpha(0), \beta(0))$ a.s. Let $a(t, x)$ and $b(t, x)$ be measurable in $G$. Suppose the following assumptions are satisfied:

1) $|a(t, x)-a(t, y)|+|b(t, x)-b(t, y)| \leq K|x-y|$ for $(t, x),(t, y) \in G$,

2) $b(t, \alpha(t))=b(t, \beta(t))=0$ for $t \in[0, T]$,

3) $a(t, \alpha(t)) \geq \alpha^{\prime}(t), a(t, \beta(t)) \leq \beta^{\prime}(t)$ for $t \in[0, T]$,

4) $\mathbb{E}\left(\Phi\left[f\left(0, X_{0}\right)\right]\right)^{2}<\infty$.

Then there exists a process $X(t)$ satisfying the conditions:

(A) $X(t)=X_{0}$ for $t=0$,

(B) $X(t) \in(\alpha(t), \beta(t))$ a.s. for $t \in[0, T]$.

(C) $\left(\Omega, \mathcal{F}, P,\left\{\mathcal{F}_{t}\right\}, W, X(t)\right)$ is a solution of $(1)$, where $\mathcal{F}_{t}=\mathcal{F}_{t}^{W} \vee \sigma\left(X_{0}\right)$.

If $X(t)$ and $\bar{X}(t)$ are two solutions of $(1)$ satisfying $(\mathrm{A})-(\mathrm{C})$, then

$$
P\left\{\sup _{0 \leq t \leq T}|X(t)-\bar{X}(t)|=0\right\}=1 .
$$

Proof. Define

$$
\begin{aligned}
a_{1}(t, x)= & \frac{\partial f}{\partial t}(t, g(t, x))+\frac{\partial f}{\partial x}(t, g(t, x)) a(t, g(t, x)) \\
& +\frac{1}{2} \frac{\partial^{2} f}{\partial x^{2}}(t, g(t, x)) b^{2}(t, g(t, x)), \\
b_{1}(t, x)= & \frac{\partial f}{\partial x}(t, g(t, x)) b(t, g(t, x)) .
\end{aligned}
$$

We will show that $a_{1}(t, x)$ and $b_{1}(t, x)$ satisfy all the assumptions of Theorem 2.

Since $f$ and $g$ are $C^{3}$, by 1$)$ the coefficients $a_{1}(t, x)$ and $b_{1}(t, x)$ satisfy Condition 1) of Theorem 2. Since $g(t,-1) \equiv \alpha(t), g(t, 1) \equiv \beta(t), f(t, \beta(t)) \equiv$ 1 and $\left.f_{x}(t, x)>0,2\right)-4$ ) imply Conditions 2$)-4$ ) of Theorem 2, respectively.

Thus, by Theorem 2, there exists a solution $X_{1}(t)$ of $(1)$ with the coefficients $a_{1}(t, x)$ and $b_{1}(t, x)$ satisfying $X_{1}(0)=f\left(0, X_{0}\right),\left|X_{1}(t)\right|<1$ a.s. for $t \in[0, T]$. In the same way as in Theorem 2 we prove that the process $X(t)=g\left(t, X_{1}(t)\right)$ is a solution of (1) with the coefficients $a(t, x)$ and $b(t, x)$. Moreover, $X(t)$ satisfies Conditions $(\mathrm{A})-(\mathrm{C})$. 
If $X(t)$ and $\bar{X}(t)$ are two solutions of (1) satisfying (A)-(C), then by Theorem 2

$$
P\left\{\sup _{0 \leq t \leq T}|X(t)-\bar{X}(t)|=0\right\}=P\left\{\sup _{0 \leq t \leq T}|f(t, X(t))-f(t, \bar{X}(t))|=0\right\}=1 .
$$

The corollary is proved.

If the conditions of Corollary 1 are fulfilled and additionally $a(t, x)$ and $b(t, x)$ are continuous in both arguments, then ([2], Theorem 2, p. 68 and $[2]$, p. 66) $X(t)$ is a diffusion with diffusion coefficient $b^{2}(t, x)$ and drift coefficient $a(t, x)$.

Acknowledgements. The author would like to thank the referee for his suggestions that have helped to generalize an earlier version of Theorem 2 .

\section{References}

[1] S. N. Ethier and T. G. Kurtz, Markov Processes. Characterization and Convergence, Wiley, New York 1986.

[2] I. I. Gikhman and A. V. Skorokhod, Stochastic Differential Equations, Springer, Berlin 1972.

[3] N. Ikeda and S. Watanabe, Stochastic Differential Equations and Diffusion Processes, North-Holland, Amsterdam 1981.

INSTITUTE OF MATHEMATICS

TECHNICAL UNIVERSITY OF CRACOW

WARSZAWSKA 24

31-155 KRAKÓW, POLAND

Reçu par la Rédaction le 20.2.1990

Révisé le 8.5.1990, 18.3.1991 et 11.10.1991 